Fundación

Miguel Lillo

Tucumán

Argentina

\title{
Aportes al conocimiento de la biología reproductiva de la Monjita Salinera (Xolmis salinarum, Passeriformes: Tyrannidae)
}

\section{Contributions to the knowledge of the reproductive biology of the 'salinas monjita' (Xolmis salinarum, Passeriformes: Tyrannidae)}

Guillermo Sferco ${ }^{1-2 *}$, Gabriel Orso ${ }^{2-3}$, Luis Urquiza², Micaela Marasas², Virginia Prelato², Agustina Salas², Guillermo Galliano², Carolina Busquetz², Juan Martínez Casas $^{2}$, Christopher Lanceley², Carlos Carmona ${ }^{2}$

1 Centro de Zoología Aplicada, Facultad de Ciencias Exactas, Físicas y Naturales, Universidad Nacional de Córdoba. Rondeau 798, (5000) Córdoba, Argentina.

2 Fundación Mil Aves. 9 de Julio 40, $2^{\circ}$ piso, of 27, (5000) Córdoba, Argentina.

3 Área Biología del comportamiento, Instituto de Investigaciones Biológicas y Tecnológicas (IIByT; CONICET-UNC) e Instituto de Ciencia y Tecnología de los Alimentos (ICTA), Facultad de Ciencias Exactas, Físicas y Naturales, Universidad Nacional de Córdoba, Córdoba, Argentina.

* Autor corresponsal: g.sferco@unc.edu.ar

\section{RESUMEN}

La Monjita Salinera es un ave endémica de ambientes salinos del centro de Argentina, de la cual existe escasa información sobre su biología y reproducción. Reportamos nuevos registros sobre la biología reproductiva de la especie, particularmente sobre un sitio de nidificación, parámetros de postura y pichones. Los registros resultaron de relevamientos en dos sitios de las Salinas Grandes de la provincia de Córdoba, de los cuales solo en uno tuvimos éxito. Observamos bandadas de entre 20 y 40 individuos en otoño e invierno, respectivamente, mientras que en primavera solo pocas parejas dispersas y tres individuos, realizando posibles despliegues de cortejo. En verano, se localizó un nido con 2 pichones, ubicado sobre el suelo en la base de un jume. Los pichones estaban cubiertos de plumón color gris perla, el pico amarillo pálido y las patas amarillentas. A los 7 días ambos habían crecido y mudado gran parte de su plumón, sin embargo uno se encontraba debilitado y el otro sin vida. Esto pudo deberse a las altas temperaturas cercanas a los $45^{\circ} \mathrm{C}$ que hubo en días

\footnotetext{
Ref. bibliográfica: Sferco, G., Orso, G., Urquiza, L., Marasas, M., Prelato, V., Salas, A., Galliano, G., Busquetz, C., Martínez Casas, J., Lanceley, C., Carmona, C. (2021). Aportes al conocimiento de la biología reproductiva de la Monjita Salinera (Xolmis salinarum, Passeriformes: Tyrannidae). Acta zoológica lilloana 65 (1): 161-172. doi: https://doi.org/10.30550/j.azl/2021.65.1/2021-03-15

> Recibido: 4 de septiembre 2020 - Aceptado: 15 de marzo 2021.

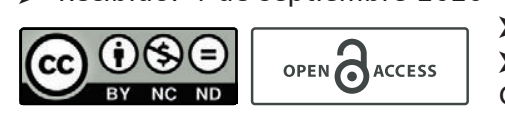

- URL de la revista: http://actazoologica.lillo.org.ar

- Esta obra está bajo una Licencia Creative Commons Atribución - No Comercial - Sin Obra Derivada 4.0 Internacional.
} 
previos. Luego del monitoreo, una lluvia de unos $90 \mathrm{~mm}$ inundó el sitio y el nido con los pichones había desaparecido al día siguiente. Se reporta por primera vez un comportamiento que podría vincularse a la competencia entre machos para cortejar una hembra, la descripción de un nido y datos de la primera muda de los pichones. Se espera que futuros estudios puedan aportar un mayor conocimiento sobre la biología reproductiva de la Monjita Salinera.

Palabras clave - Aves, Córdoba, Endemismo, Salinas Grandes.

\section{ABSTRACT}

The 'salinas monjita' is an endemic bird of the saline environments from central Argentina and there is little information about its biology and reproduction. We report new records on the reproductive biology of the species, particularly on a nesting site, posture parameters and chicks. The records resulted from surveys at sites in Salinas Grandes salt flats in the province of Córdoba, of which we were successful in only one. We observed flocks of between 20 and 40 individuals in autumn and winter, respectively, while in spring only a few scattered pairs and three individuals, performing possible courtship displays. In the summer, a nest with two chicks was located on the ground at the base of a jume. The chicks were covered with pearl grey down, the beak was pale yellow and the legs were yellowish. After seven days, both had grown and molted much of their down, but one was weak and the other lifeless. This may have been due to the high temperatures of $45^{\circ} \mathrm{C}$ in the previous days. After monitoring, a $90 \mathrm{~mm}$ rain shower flooded the site and the nest with the chicks had disappeared the next day. For the first time, we reported a behavior that could be linked to competition between males to court a female, description of a nest and data on the nestlings first molt. It is hoped that future studies can provide greater knowledge about the reproductive biology of the salinas monjita.

Keywords - Birds, Córdoba, Endemism, Salinas Grandes.

\section{INTRODUCCIÓN}

La Monjita Salinera (Xolmis salinarum) es una especie de aproximadamente $17 \mathrm{~cm}$ de largo, que pertenece a la familia Tyrannidae. Es endémica del centro de Argentina, restringiendo su distribución a ambientes salinos de las provincias de Córdoba, Santiago del Estero, Catamarca y La Rioja (Farnsworth, Langham, Boesman, 2020). Aunque en las últimas dos décadas se han sumado algunos registros en las provincias de San Luis (Nellar, 1993; Haene, 2005; Chebez, 2009), San Juan (Martínez et al., 2009; Gelain y Pereyra Lobos, 2011; Lucero, 2013a y 2013b), Mendoza (Martínez et al., 2009; Lucero, 2013a) y Salta (Moschione, Spitznagel, González, 2013).

Esta especie fue descripta por primera vez por Nores e Yzurieta (1979), quienes la consideraron como subespecie de la Monjita Castaña (Xolmis rubetra). Si bien en un primer momento algunos autores sostenían que no existen diferencias para elevarla a nivel de especie (Nores, 1996; Stotz, Fitzpatrick, Parker III, Moskovits, 1996), 
actualmente existe consenso científico para considerar a $X$. rubetra y $X$. salinarum como especies diferentes. Este consenso se basa en la existencia de diferencias en el tamaño de las aves, el plumaje de adultos y de pichones, migración, comportamiento y hábitat (Olrog, 1984; Narosky e Yzurieta, 1987; Canevari, et al., 1991; Mazar Barnett y Pearman, 2001; Remsen et al., 2020).

El hábitat que más frecuenta la Monjita Salinera es el Matorral Halófito Bajo de Allenrolfea patagonica y Heterostachys ritteriana (Jumes), que comprende vegetación arbustiva halófita en los límites de las playas salinas con un estrato herbáceo ausente o muy reducido. El alejamiento de los bordes salinos y las elevaciones del terreno favorecen la aparición de otros elementos vegetales, como el Jume o Vinagrillo ( $\mathrm{Sa}$ licornia ambigua) o arbustos espinosos como el Cachiyuyo (Atriplex argentina), Zampa (Atriplex lampa), Mastuerzo o Retortuño (Prosopis reptans), entre los más característicos (Cabido y Zak, 1999). Ocasionalmente, la Monjita Salinera puede alcanzar los bordes del bosque chaqueño, en el ecotono con las salinas (Remsen et al., 2020; Farnsworth et al., 2020).

Se considera a la Monjita Salinera como una especie de poco a bastante común o frecuente (Nores, 1996; Salvador, Salvador, Ferrari, Vitale, 2016; Farnsworth $e t$ $a l ., 2020)$. Generalmente se la observa posada en sitios visibles o corriendo por el suelo en busca de alimento. Durante la época reproductiva se la observa en parejas dispersas, mientras que en invierno se agrupa en bandadas que pueden llegar a contabilizar hasta 40 individuos (Remsen et al., 2020; obs. pers.). En reposo sexual se cree que pueda efectuar algunos desplazamientos, ya que ha sido hallada ocasionalmente en Salta en esta época (Moschione, Spitznagel y González, 2012).

Globalmente se la clasifica como Cercana a la Amenaza, y a nivel nacional como Vulnerable (Ministerio de Ambiente y Desarrollo Sustentable y Aves Argentinas, 2017; BirdLife International, 2020). Si bien se indica que su población se encontraría estable, su número poblacional es desconocido, así como gran parte de su biología en general. En cuanto a su biología reproductiva en particular, existe un único registro documentado donde se describen un nido con 1 huevo y 1 pichón, 3 nidos vacíos, los meses de comienzo de reproducción, el cortejo y la crianza de los pichones y juveniles de la Monjita Salinera (Cobos y Miatello, 2011). Por otra parte, existe un registro fotográfico del año 2017 en San José de las Salinas (provincia de Córdoba) de un nido con 2 huevos construido sobre el suelo (Uranga, 2017), y otro del año 2020, en el mismo sitio, de un adulto alimentando a un joven (Caballero, 2020).

Debido a la falta de conocimiento general de la especie y más aún a las ciertas características de su reproducción, nos propusimos como objetivo: estudiar la biología reproductiva de la Monjita Salinera, particularmente el lugar donde ubican los nidos, parámetros de postura de huevos y de sus pichones, a través de relevamientos de dos sitios de las Salinas Grandes de la provincia de Córdoba.

\section{MATERIAL Y MÉTODOS}

El sitio de estudio se ubica en la Reserva de Usos Múltiples Salinas Grandes, al noroeste de la provincia de Córdoba. La Reserva cuenta con 190.000 ha, y dentro 
de ella se encuentra también el Refugio de Vida Silvestre Monte Las Barrancas de 7.656 ha. Esta última área protegida es una elevación sobre la superficie de las salinas, con barrancas en el oeste de hasta $8 \mathrm{~m}$ de altura. El gradiente de salinidad entre las tierras elevadas y bajas determina una sucesión que va desde un bosque Chaqueño Árido en las barrancas, hasta la vegetación halófita de la estepa salina (Figura 1). Es por ello que el Refugio de Vida Silvestre Monte las Barrancas se considera una "isla boscosa" dentro de una matriz de salinas. Las precipitaciones son irregulares espacialmente y concentradas entre los meses de noviembre y marzo (Contreras, Coirini, Karlin, Ruiz Posse, 2010), promediando anualmente entre 300 y $492 \mathrm{~mm}$, que suelen inundar temporariamente las zonas más bajas. La temperatura media anual ronda los $21^{\circ} \mathrm{C}$, registrándose máximas de $50^{\circ} \mathrm{C}$.

Sobre la base de la información y de relevamientos previos, realizamos un total de 11 campañas en dos de los sitios de mayor probabilidad de ocurrencia de la especie. Uno de estos sitios se localiza a aproximadamente $2.5 \mathrm{~km}$ al este de la localidad de San José de las Salinas, y fue visitado en tres oportunidades: junio y julio de 2018, y julio de 2019. El otro sitio está ubicado a $5 \mathrm{~km}$ al noroeste del paraje Las Toscas, y fue visitado en ocho oportunidades entre febrero de 2019 y enero de 2020.

Cada campaña abarcó 1 día de muestreo, comenzando a las 06:00 h y finalizando a las 17:00 h, aproximadamente. En las campañas participaron cinco observadores,

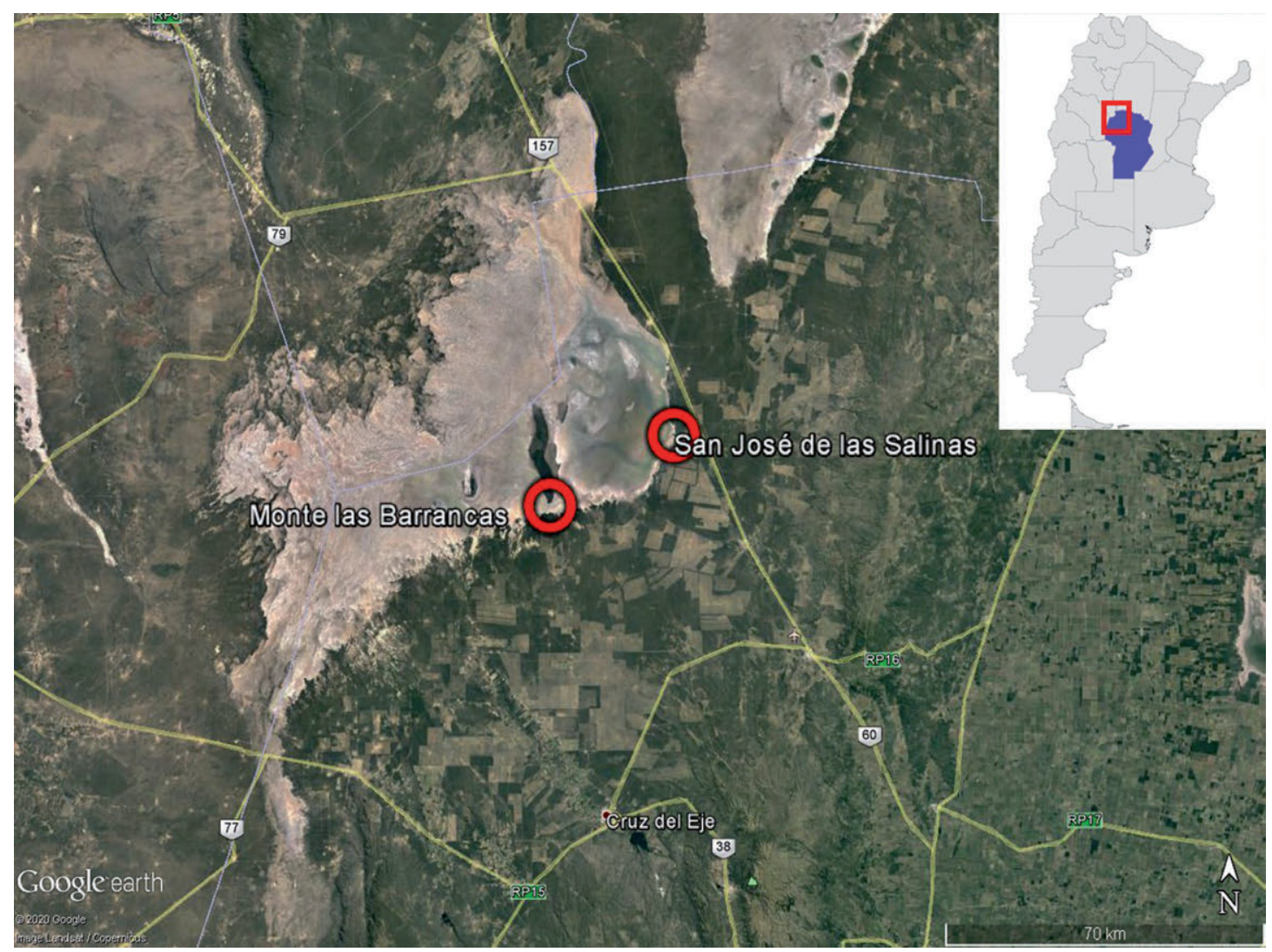

Figura 1. Ubicación geográfica de los sitios donde se realizaron las campañas. El recuadro rojo indica la ubicación de las Salinas Grandes y los círculos rojos los sitios de relevamiento.

Figure 1. Geographical location of the sites where the campaigns were carried out. The red box indicates the location of the Salinas Grandes and the red circles the survey sites. 
los cuales se desplazaban por transectas paralelas de entre 2 y $3 \mathrm{Km}$ de largo, separadas por $10 \mathrm{~m}$ y distribuidas en el gradiente entre la vegetación arbustiva halófita y la playa de la salina, que son las áreas de mayor probabilidad de encuentro con la especie. Para detectar los sitios de nidificación se siguió con binoculares a individuos acarreando material para la construcción del nido o llevando alimento en su pico por espacio de varios minutos, lo cual indicaría que se encuentra construyendo el nido o alimentando a los pichones (Ralph et al., 1996).

\section{RESULTADOS}

En los tres relevamientos realizados en San José de las Salinas no se detectó ningún ejemplar, por lo que se concentró el esfuerzo de muestreo en el Refugio de Vida Silvestre Monte las Barrancas, donde tuvimos encuentro con la especie en todas las visitas. En este último sitio se registraron bandadas de 20 ejemplares en junio de 2019 y de 40 ejemplares en julio del mismo año. Las bandadas no eran compactas y los individuos se situaban entre 3 y $10 \mathrm{~m}$ de distancia entre ellos. Realizaban vuelos cortos y bajos alternando con recorridos en el suelo, posándose cada tanto en los arbustos.

En las seis campañas de primavera y verano, se observaron tres parejas en noviembre de 2019, separadas entre sí entre 150 y 300 m y el 3 de diciembre de 2019 se registraron tres individuos. Uno de ellos realizaba vuelos a gran velocidad en dirección hacia otro, y viceversa. Ambos individuos que interactuaban, además desplegaban vuelos verticales con las alas abiertas y el dorso dirigido hacia el otro individuo, para luego posarse en un jume. Mientras tanto el tercer individuo permaneció en el suelo.

El 22 de diciembre de 2019 se observó solo una pareja y detectamos que uno de los ejemplares tenía alimento en el pico e ingresó en la base de un arbusto. Allí observamos un nido de Monjita Salinera con 2 pichones, que se encontraba sobre el suelo, semiexpuesto en la base de un jume (Salicornia sp.), en el límite de vegetación antes que comience la estepa salina (3009'2,6" S; 64'54'13,4” W). La altura del jume era de $50 \mathrm{~cm}$ y se encontraba rodeado por otros jumes a distancias de 60, 85 y $160 \mathrm{~cm}$ (Figura 2). El nido estaba construido mayormente con tallos y fibras vegetales, tenía forma de taza y medía $14.5 \mathrm{~cm}$ de diámetro externo, $7.5 \mathrm{~cm}$ de diámetro interno y $3.4 \mathrm{~cm}$ de profundidad. Ambos pichones medían $5.5 \mathrm{~cm}$ de pico a cola, presentaban los ojos cerrados y se encontraban cubiertos de un plumón color gris perla. Tenían el pico de color amarillo pálido con el borde amarillo más intenso y el interior de la boca rojo, mientras que las patas eran de color amarillento (Figura 3).

A los 7 días posteriores al hallazgo, pudimos detectar a un adulto a menos de $10 \mathrm{~m}$ del nido. Constatamos que ambos pichones habían crecido y medían $8 \mathrm{~cm}$ de pico a cola, y uno de ellos había mudado la mayor parte de su plumón de la zona de la cabeza, dorso, remeras primarias y secundarias y cobertoras hacia un plumaje juvenil o primer básico, con plumas pardas y castañas, presentaba los ojos abiertos, se encontraba inmóvil la mayor parte del tiempo y cuando realizaba algún movimiento, lo hacía con lentitud. El otro pichón se hallaba sin vida, mostraba signos de muda en las mismas zonas que el otro, pero aún conservaba algo de plumón (Figura 4). 


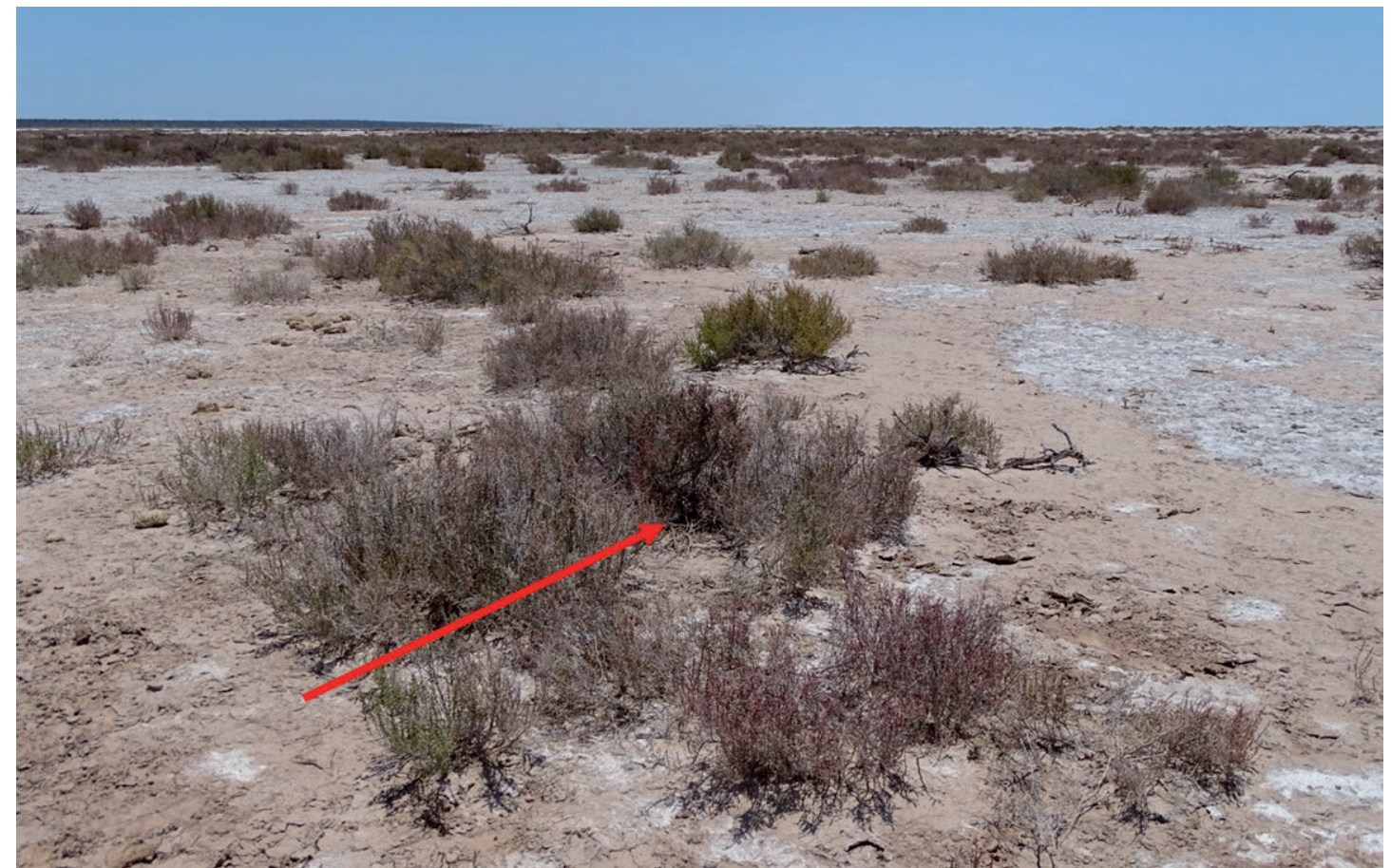

Figura 2. Ambiente y ubicación del nido de Xolmis salinarum con dos pichones. Fotografía: Guillermo Sferco.

Figure 2. Environment and location of the nest of Xolmis salinarum with two chicks. Photo: Guillermo Sferco.

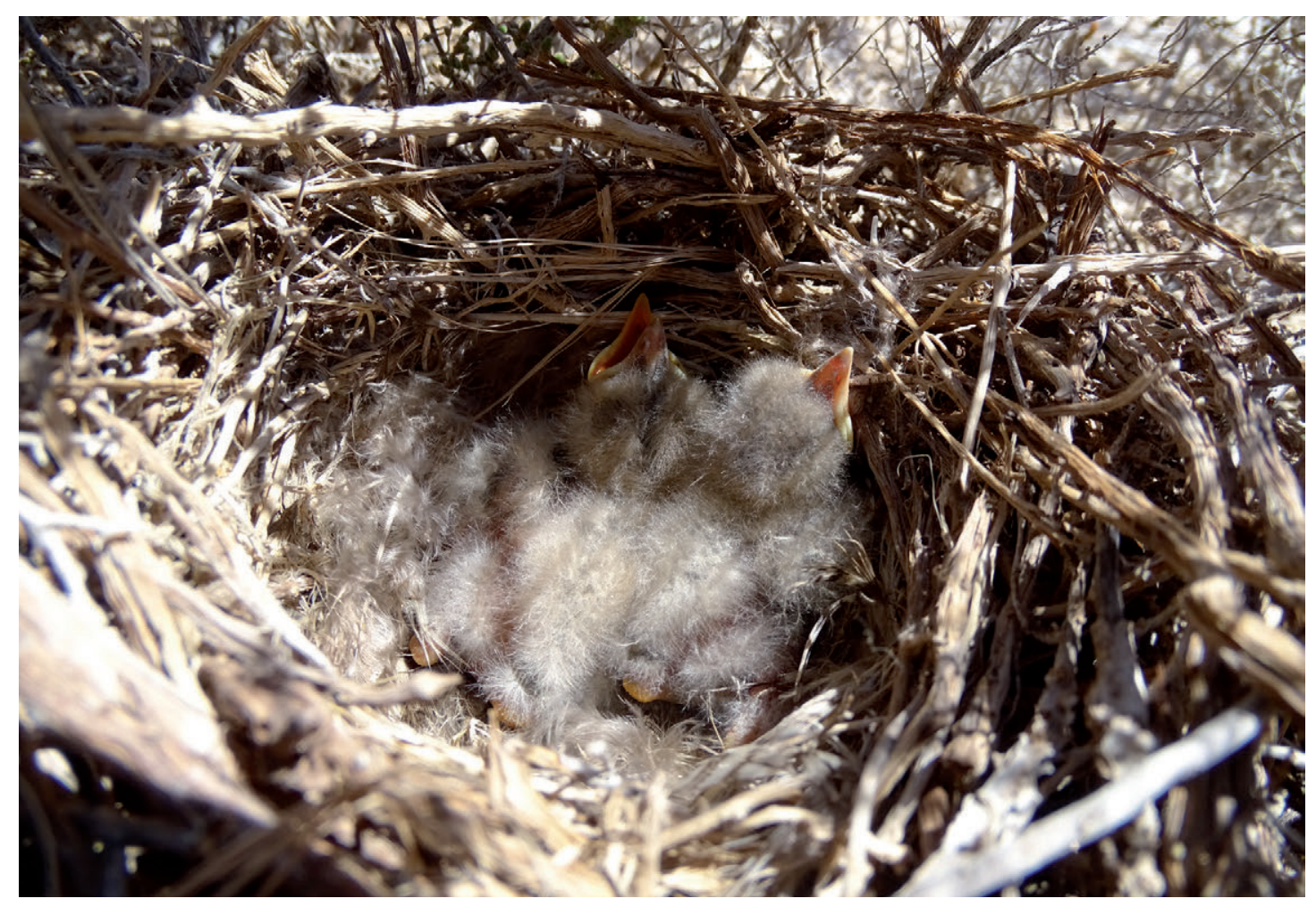

Figura 3. Pichones de Xolmis salinarum, donde se aprecia el color del plumón, picos y patas. Fotografía: Guillermo Sferco.

Figure 3. Xolmis salinarum chicks, showing the color of the down, beaks and legs. Photo: Guillermo Sferco. 


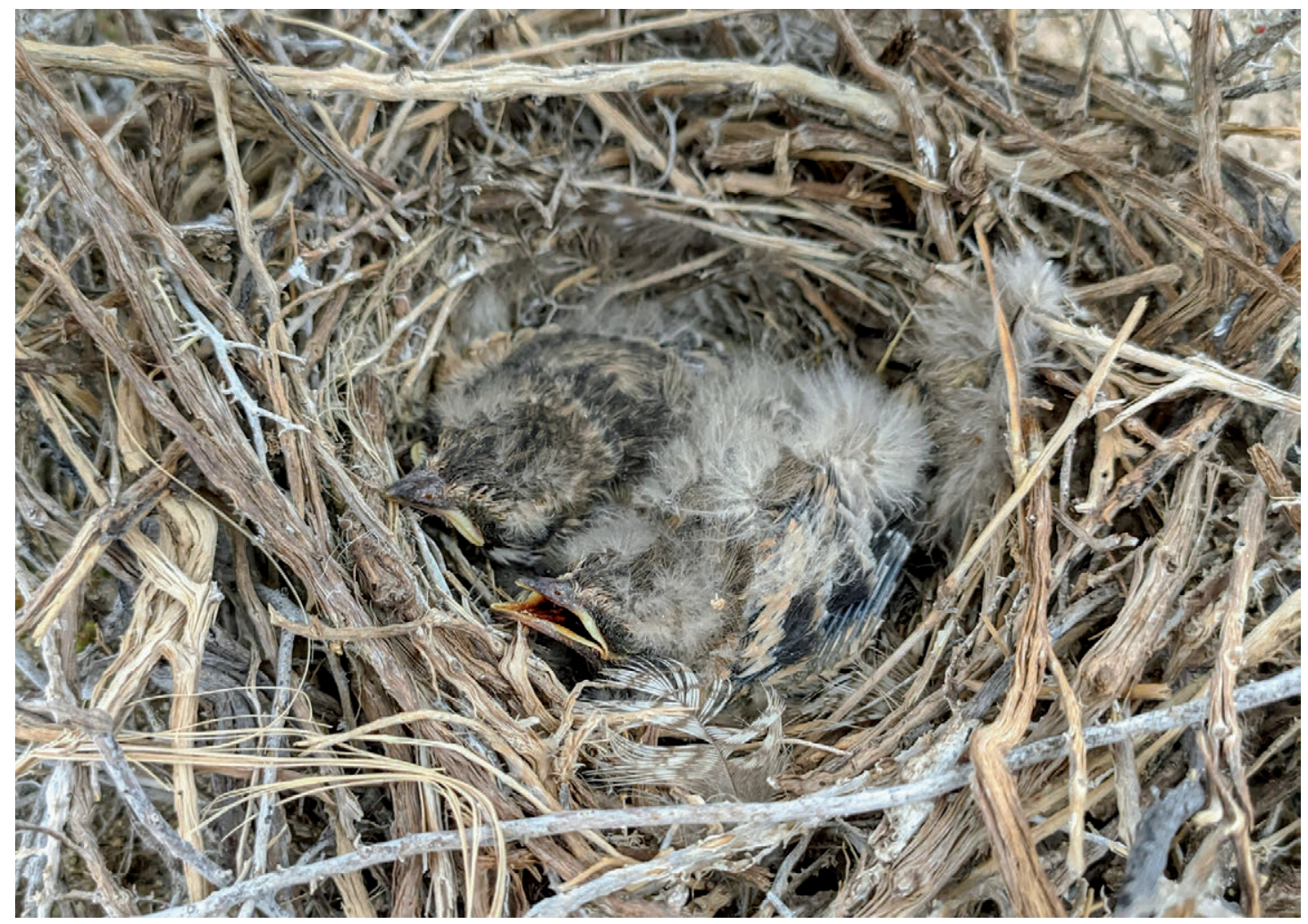

Figura 4. Pichones de Xolmis salinarum a los siete días del hallazgo. El de la derecha, con más plumón, se hallaba sin vida. Fotografía: Gabriel Orso.

Figure 4. Xolmis salinarum chicks seven days after being found. The one on the right, with more down, was lifeless. Photo: Gabriel Orso.

Luego de esta visita hubo una gran tormenta donde precipitaron aproximadamente $90 \mathrm{~mm}$ de lluvia, y como consecuencia se inundó el suelo salino. Comprobamos que la zona en donde se encontraba el nido estaba completamente anegada, formándose incluso corrientes de agua que bajaban por las barrancas y se dirigían hacia la estepa salina. No se logró hallar el nido, presumiblemente porque se encontraría bajo el agua o habría sido desplazado por la corriente, por lo que asumimos que el pichón restante también murió.

La última campaña se realizó el día 26 de enero de 2020. Se detectó la presencia de dos adultos de Monjita Salinera a una distancia de $30 \mathrm{~m}$ de un nido vacío, que por su tamaño y materiales de construcción, pertenecería a esta especie. El mismo se encontraba a $30 \mathrm{~cm}$ del suelo, oculto dentro de un jume (Figura 5).

\section{DISCUSIÓN}

En primavera se observó la formación de un trío de Monjita Salinera en el que dos de los individuos interactuaban, mientras que el restante permanecía en el suelo forrajeando. Nuestras observaciones se encuentran en concordancia con la idea de que el comportamiento registrado podría ser utilizado por los machos de la Monjita Salinera para cortejar a la hembra y/o competir con otros machos. Sin embargo, 


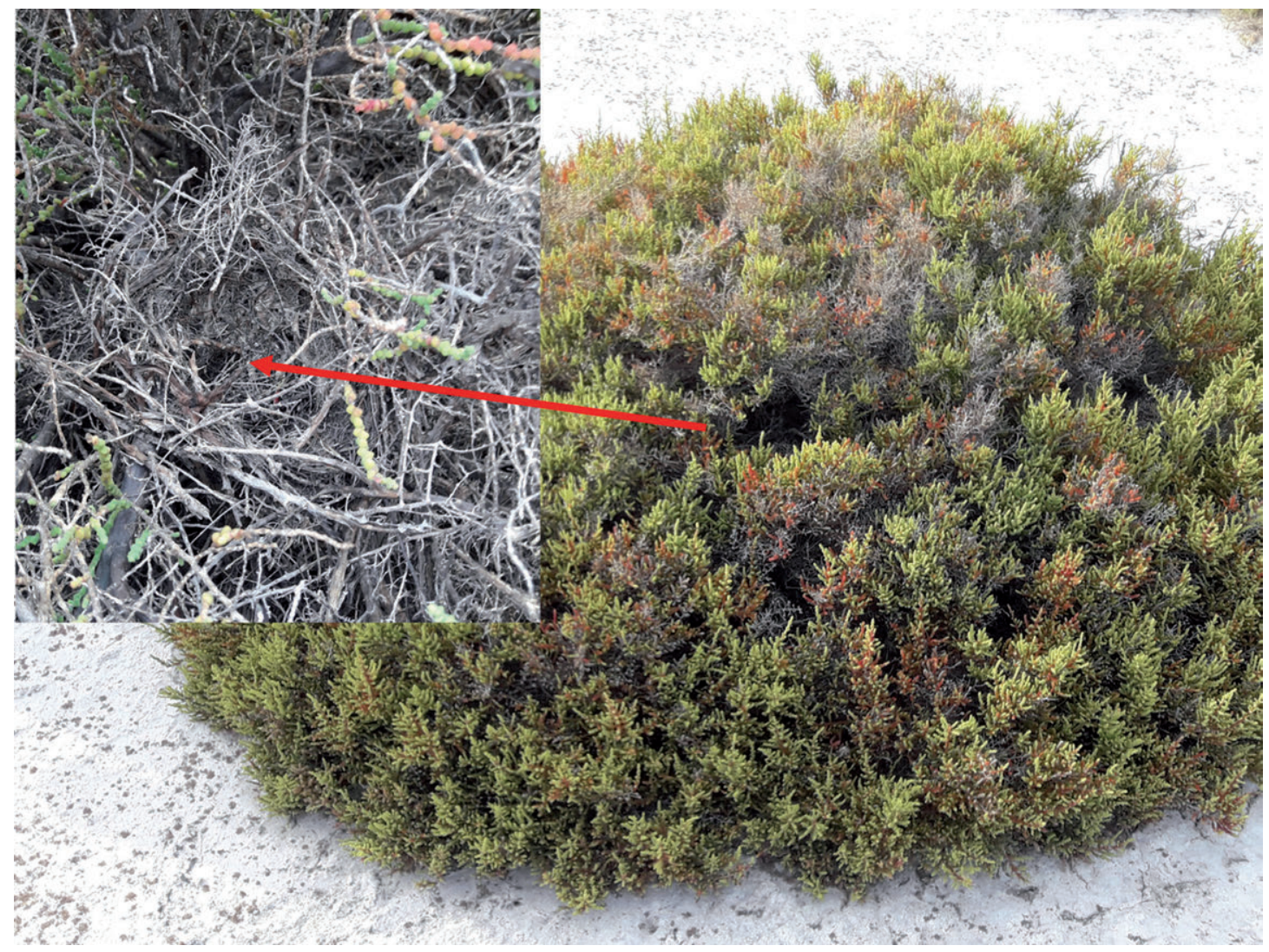

Figura 5. Nido abandonado, atribuible a Xolmis salinarum. Fotografía: Carlos Carmona.

Figure 5. Abandoned nest, attributable to Xolmis salinarum. Photo: Carlos Carmona

Cobos y Miatello (2001) describen una interacción entre parejas similar al observado por nosotros, aunque mencionan un solo individuo realizando los despliegues y lo atribuyen a un cortejo.

El nido activo reportado en este trabajo, se ubicaba en la base de un jume, sobre el suelo, coincidente con el registro fotográfico realizado por Uranga (2017). Sin embargo, el nido abandonado que se registró en el último muestreo se encontraba a unos $30 \mathrm{~cm}$ del suelo, en similar ubicación a los 4 nidos (1 nido activo y 3 nidos vacíos) reportados por Cobos y Miatello (2001). Estos autores sugieren que la altura a la que se encuentra el nido podría ser un carácter diagnóstico de la Monjita Castaña y la Monjita Salinera, ya que la primera lo construye sobre el suelo (Vuilleumier 1994) y la segunda en altura, posiblemente debido al sustrato barroso de las salinas en época de lluvia y a la disponibilidad de arbustos en la zona (Cobos y Miatello, 2001). Sin embargo, teniendo en cuenta todos los registros, podríamos estar en presencia de una variabilidad intraespecífica de estrategia de construcción de nido en la Monjita Salinera.

El hallazgo del nido con pichones de Monjita Salinera es el segundo documentado en la literatura, de allí la importancia de la descripción realizada en el presente estudio. Nuestra descripción de la coloración del plumón como gris perla coincide con la realizada por Cobos y Miatello (2001). Sin embargo, los autores señalan que el pico era de color amarillento con la comisura blancuzca y el interior del mismo 
anaranjado, mientras que los pichones aquí descriptos presentaban el pico de color amarillo pálido con el borde amarillo más intenso y el interior de la boca rojo. Además, el color de las patas de los pichones del presente estudio era amarillento y Cobos y Miatello (2001) las describen de color rosáceo. Esto puede deberse a diferencias individuales de cada observador en la percepción del color o a descripciones de distintas etapas de desarrollo de las aves, ya que una semana después del hallazgo los pichones de este estudio habían mudado parte de su plumón por plumas pardas y castañas, y presentaban el pico de color marrón oscuro con los bordes naranja virando a amarrillo claro en la comisura. Estas descripciones son de considerable importancia debido a que la coloración del plumón y del pico son tenidas en cuenta como caracteres diagnóstico para justificar la separación de la Monjita Salinera y la Monjita Castaña en dos especies diferentes (SACC, 2008).

La muerte de uno de los pichones y la baja actividad del otro podría deberse a las altas temperaturas máximas registradas en esos días (entre $35^{\circ} \mathrm{C}$ y $45^{\circ} \mathrm{C}$ ). A su vez, la violenta tormenta y la consiguiente inundación del área, provocó la desaparición del nido y la probable muerte del pichón restante. Si bien pueden existir diferentes causas por las cuales una pareja abandone un nido en actividad, no creemos que este haya sido el caso. Como reportamos, en los encuentros constatamos la presencia de uno o dos adultos en cercanías del nido y los pichones habían crecido de tamaño y habían mudado el plumaje, lo cual significa que durante el período de nuestras observaciones, existió cuidado parental. Las características ambientales y climáticas extremas donde ocurre la Monjita Salinera (Contreras, Coirini, Karlin, Ruiz Posse, 2010) podrían eventualmente afectar negativamente el éxito reproductivo de la especie. Nuestro aporte sobre algunos aspectos de la biología reproductiva de la Monjita Salinera, resulta de considerable importancia para una especie endémica de la que se posee escasa información. Se reporta por primera vez la interacción entre 3 individuos, que podría vincularse a la competencia entre machos o un cortejo de una hembra, la descripción de un nido y datos de la primera muda de los pichones. No obstante, las observaciones que pudimos aportar resultan insuficientes para poder definir la estrategia reproductiva en esta especie. Se espera que futuros estudios puedan aportar un mayor conocimiento sobre la biología reproductiva de la Monjita Salinera, que pueda ser útil para la conservación de la especie.

\section{AGRADECIMIENTOS}

Queremos agradecer a Germán Chávez, responsable de las Áreas Naturales Protegidas de la provincia de Córdoba, por facilitarnos los permisos correspondientes de investigación en el lugar y a Nicolás Maldonado, Guardaparque de Monte las Barrancas por asistirnos en las tareas de campaña. Revisores anónimos y el editor, mejoraron sustancialmente el manuscrito. 


\section{FINANCIAMIENTO}

Todas las campañas fueron solventadas por la Fundación Mil Aves.

\section{PARTICIPACIÓN}

G. Sferco y G. Orso coordinaron el proyecto, redactaron el manuscrito y participaron en las campañas de toma de datos. L. Urquiza encontró el nido junto a M. Marasas, V. Prelato y A. Salas, también participaron en algunas campañas. G. Galliano coordinó los viajes y participó de las observaciones. C. Busquetz participó de las observaciones. J. Martínez Casas, C. Carmona y G. Galliano tomaron fotografías y realizaron observaciones, $\mathrm{Ch}$. Lanceley participó de las observaciones y asistencia en campo.

\section{CONFLICTOS DE INTERÉS}

No existe ningún conflicto de intereses entre los autores.

\section{BIBLIOGRAFÍA}

BirdLife International. (2020). Species factsheet: Xolmis salinarum. URL: http://datazone.birdlife.org/species/factsheet/salinas-monjita-xolmis-salinarum/text. Accedido en febrero del 2020.

Caballero, L. (2020). Ecoregistros. URL: http://www.ecoregistros.org/site/imagen. php?id=384755. Accedido en febrero del 2020.

Cabido, M., Zak, M. (1999). La vegetación del norte de Córdoba. Secretaría de Agricultura y Recursos Renovables de la Provincia de Córdoba. Agencia Córdoba Ambiente, $56 \mathrm{pp}$.

Canevari, M., Canevari, P, Carrizo, G., Harris, G., Rodriguez Mata, J., Straneck, R. (1991). Nueva guía de las aves argentinas. Fundación Acindar. Buenos Aires, Argentina.

Chebez, J. C. (2009). Otros que se van: fauna argentina amenazada. Buenos Aires, Argentina: Albatros.

Cobos, V., Miatello, R. (2001). Description of the nest, egg, and chick of the Salinas Monjita (Neoxolmis salinarum). El Hornero, 16, 47-48.

Contreras, A., Coirini, R., Karlin, M., Ruiz Posse, E. (2010). Clima regional y local. En Manejo sustentable del ecosistema Salinas Grandes, Chaco Árido (63-70). Coirini, R.; Karlin, M. y G. Reati Editores Literarios. Encuentro grupo editor. Córdoba, Argentina.

Farnsworth, A., Langham, G., Boesman, P. (2020). Salinas Monjita (Xolmis salinarum). En del Hoyo, J., Elliott, A., Sargatal, J., Christie, D. A., de Juana, E. (eds.). Handbook of the Birds of the World Alive. Lynx Edicions, Barcelona. URL: https://www.hbw.com/node/57429. Accedido en febrero de 2020. 
Gelain, M. A. y R. Pereyra Lobos. (2011). Lista de aves de la provincia de San Juan, Argentina. Xolmis CRO 3: 1-12.

Haene, E. H. 2005. Parque Nacional Sierra de las Quijadas. En A. S. Di Giacomo (editor). Áreas importantes para la conservación de las aves en Argentina. Sitios prioritarios para la conservación de la biodiversidad: 400-401. Temas de Naturaleza y Conservación 5. Aves Argentinas/Asociación Ornitológica del Plata. Buenos Aires.

Handbook of the Birds of the World and BirdLife International. (2019). Handbook of the Birds of the World and BirdLife International digital checklist of the birds of the world. Version 4. URL: http://datazone.birdlife.org/userfiles/file/ Species/Taxonomy/HBW-BirdLife_Checklist_v4_Dec19.zip. Accedido en febrero del 2020.

Lucero, F. (2013a). Listado de las aves observadas en la Reserva Natural Municipal Articulada: Laguna Guanacache, Laguna del Toro y Bañados del Carau, provincia de San Juan y Laguna Seca y los Bañados del Tulumaya, provincia de Mendoza, Argentina. Nótulas Faunísticas (segunda serie), 137: 1-16. Buenos Aires, Argentina.

Lucero, F. (2013b). Aves de Sarmiento, provincia de San Juan. Edición del autor. $1^{\text {a }}$ Ed., Cienaguita, 334 págs.

Martínez, F., Lucero, F., Calí, R., Valdés, D., Ferrer, D., Chebez, J. C. (2009). Registros novedosos de aves para las provincias de Mendoza y San Juan. Nótulas Faunísticas (segunda serie), 35, 1-9.

Mazar Barnett, J., Pearman, M. (2001). Lista comentada de las aves argentinas: annotated checklist of the birds of Argentina. Lynx Editions. Barcelona, España.

Ministerio de Ambiente y Desarrollo Sustentable y Aves Argentinas. (2017). Categorización de las Aves de la Argentina (2015). Informe del Ministerio de Ambiente y Desarrollo Sustentable de la Nación y de Aves Argentinas, edición electrónica. C. A. Buenos Aires, Argentina. 147 pp

Moschione, F., Spitznagel, O., González, M. (2012). Lista de aves de Salta (Birds checklist). Gobierno de la Provincia de Salta.

Narosky, T., Yzurieta, D. (1987). Guía para la identificación de las aves de Argentina y Uruguay. Buenos Aires: Vazquez - Mazzini Editores.

Nellar Ramonella, M. M. (1993). Aves de la provincia de San Luis. Lista y distribución. Ed. Mus. Priv. Cienc. Nat. e Inv. Ornit. “G. E. Hudson”. San Luis. Págs. 98.

Nores, M. (1996). Avifauna de la provincia de Córdoba. Biodiversidad de la provincia de Córdoba. Fauna, 1, 255-337.

Nores, M., Yzurieta, D. (1979). Una nueva especie y dos nuevas subespecies de aves (Passeriformes). Academia Nacional de Ciencias de Córdoba, Miscelánea, 61, 4-8.

Olrog, C. C. (1984). Las aves argentinas. Una nueva guía de campo. Buenos Aires: Administración de Parques Nacionales.

Ralph, C., Geupel, G., Pyle, P., Martin, T. E., De Sante, D., Mila, B. (1996). Manual de métodos de campo para el monitoreo de aves terrestres. Gen. Tech. Rep. 
PSW-GTR-159. Albany, CA: Pacific Southwest Research Station, Forest Service, U.S. Department of Agriculture, $46 \mathrm{pp}$.

Remsen, J. V., Jr., Areta, J. I., Bonaccorso, E., Claramunt, S., Jaramillo, A., Pacheco, J. F., Ribas, C., Robbins, M. B., Stiles, F. G., Stotz, D. F., Zimmer, K. J. (2020). A classification of the bird species of South America. American Ornithological Society. URL: http://www.museum.lsu.edu/ Remsen/SACCprop350.htm.

Salvador, S. A., Salvador, L. A., Ferrari, C., Vitale, S. (2016). Listado de aves de la provincia de Córdoba, Argentina. Buenos Aires: Aves Argentinas.

South American Classification Committee (SACC). (2008). Proposal (\#350) to South American Classification Committee: incluir Xolmis salinarum dentro de X. rubetra. URL: http://www.museum.lsu.edu/ Remsen/SACCprop350.htm. Accedido en febrero de 2020.

Stotz, D. F., Fitzpatrick, J. W., Parker III, T. A., Moskovits, D. K. (1996). Neotropical birds. Ecology and conservation. Chicago, IL: University of Chicago Press.

Uranga, M. (2017). Ecoregistros. URL: http://www.ecoregistros.org/site/imagen. php?id=236009. Accedido en febrero de 2020.

Vuilleumier, F. (1994). Nesting, behavior, distribution, and speciation of Patagonian and Andean ground tyrants (Myotheretes, Xolmis, Neoxolmis, Agriornis, and Muscisaxicola). Ornitología Neotropical, 5, 1-55. 